\title{
Interactive comment on "Can morphological features of coccolithophores serve as a reliable proxy to reconstruct environmental conditions of the past?" by Giulia Faucher et al.
}

\section{Giulia Faucher et al. \\ giulia.faucher@unimi.it}

Received and published: 16 December 2019

We thank the anonymous reviewer for the helpful comments on our manuscript. The reviewer also provided several editorial suggestions, which we all considered in our revision of the text. Below we focused our discussion on the more topical points raised by the reviewer.

With regards Giulia Faucher

1) RC: I suggest that the authors should explain more about why they design these tests on coccolithophore in the Introduction or Methods part, such as the carbonic 
chemistry experiments are for ocean acidification and $\mathrm{Mg} / \mathrm{Ca}$ experiments are for the long-time evolution of seawater. How about considering move the first paragraph in discussion into introduction? Because I feel that I am clearer for the study motivation after reading

AC: We thank the referee for the suggestion: we discuss a lot on the introduction and we tweaked it to make the motivation of our work clearer. However, we decided to leave the details about the paleo-record out of this chapter. Indeed, we think that a short introduction is more effective to the reader. We hope that the reviewer agrees on our idea of living the text fully tailored towards the primary goal of our experiments, and aligned the introduction with the title. Finally, the paleo-context is carefully considered in the discussion.

2) RC: I doubt about can the timing of measurement influence the coccolith length results. Because a previous work shows the growth phase can influence the coccolith and cell size (Sheward et al., 2017). That also means if the cells are harvested right after and before dividing, the results should be different. Since there were light-dark cycles in your experiment setting, the authors should mention when they harvest the cells for size measurement and are these timings same among different experiment.

AC: We thank the referee for suggesting us to read and take into consideration the very interesting paper by Sheward et al., (2017). This study demonstrated that the coccosphere, cell size and coccolith number of the analyzed species are influenced by the growth phase that the algae are experiencing. Coccolith sizes don't have any direct link with coccosphere diameters or growth stages. We underline that in our experiments, to avoid an artifact in our data, all measurements were started and conducted at the same time for every experiment that we performed. This information was added to the text.

3) RC:I did not find how many coccoliths did they measured in the Methods part, which is important for statistical analyses. 
AC: We measured 50 specimens for every treatment and every replicate. This information was highlighted in the text and in the caption of the figures (see 2.3).

4) RC: In Figure 2, does the ' $C$ ' mean 'control experiments'. If so, why the results in different control experiment are quite different? Are the experiment settings in different control experiments different or the same? If the settings are same, why the results so different?

AC: It was added in the caption that $C$ means control conditions. When we performed the experiment, we decided to investigate the same parameter simultaneously for all the tested species. But, the 5 parameters were tested in different experiments performed in a row at different times (different months and years). As indicate by Langer et al., (2012), the morphology of a strain that grew under similar conditions can change over time. The author highlighted, however, that these changes occur at a timescale that is longer of a typical duration of a culture experiment. That means that the experiment validity is not impaired, however, every experiment needs to be considered by itself.

Langer, G., Oetjen, K., \& Brenneis, T. (2013). On culture artifacts in coccolith morphology. Helgoland Marine Research, 67(2), 359.

5) RC: I did not find where they discuss the results of ellipticity, rays, bridge angles. That is why I say they should dig their data deeper.

AC: The referee is right. We measured many parameters due to different morphologies of the tested species (e.g. E. huxleyi distal shield element average number, G. oceanica bridge angle). The data are represented in tables for every experiment. However, these morphological features are species-specific and it would have not been possible to compare to metrics for the other coccolithophores. The comparison among different species was the main goal of our study. Therefore, we think that it makes more sense to consider morphological features that can be measured for all coccolithophores (e.g. coccolith size). 
6) RC: They found that different coccolithophores have different response to the environment settings. From my point of view, that is not something surprises me: they should not behave in a similar way. What we really want to know is why is that, so the authors should try to offer an explanation rather than leaving the conclusion in this way. In this case, I do not quite agree with the opinion that coccolith morphological data can only serve as a paleo-proxy when different species have a same response to the environment variations. Well, that is only my idea, and the authors do not have to change their conclusion if they can offer a better discussion than this version.

AC: We agree with referee1: it is indeed not surprising that morphology responds differently among species to changing environmental conditions. This was also our hypothesis before we conducted the experiments. However, this assumption (conserved response over geological timescales) is frequently made in studies that investigate responses in living coccolithophores to explain paleo-responses. Therefore, we tested if this frequently made assumptions are justifiable. Furthermore, we were interested if there is something like a "universal response" at least to some of the tested environmental parameters. Such a universal response among evolutionarily very distinct coccolithophores could suggest that the reaction is "conserved" for geological timescales and would improve our confidence that morphology could be used as a paleo-proxy. We only find such a rather universal response for the malformation response to carbonate chemistry. In this regard, we thank the referee for suggesting us to take into consideration the paper by Aloisi, (2015). In this paper, the author suggests that the environment controls coccolith sizes (and or morphology) via a physiological effect and for that reason, he managed to build a model that simulates growth rates and sizes of cells, applicable to the whole coccolithophore community.

7) AC: These two papers may help for a rich discussion, Sheward et al. (2017) and Aloisi (2015), which are absent from their references list.

Printer-friendly version

Discussion paper

References:

Interactive

comment 
Sheward R M, Poulton A J, Gibbs S J, et al. Physiology regulates the relationship between coccosphere geometry and growth phase in coccolithophores[J]. Biogeosciences, 2017, 14(6): 1493-1509.

Aloisi G. Covariation of metabolic rates and cell size in coccolithophores[J]. 2015

AC: We thank the referee for the suggestions. The two papers were considered.

Interactive

comment

Interactive comment on Clim. Past Discuss., https://doi.org/10.5194/cp-2019-84, 2019. 